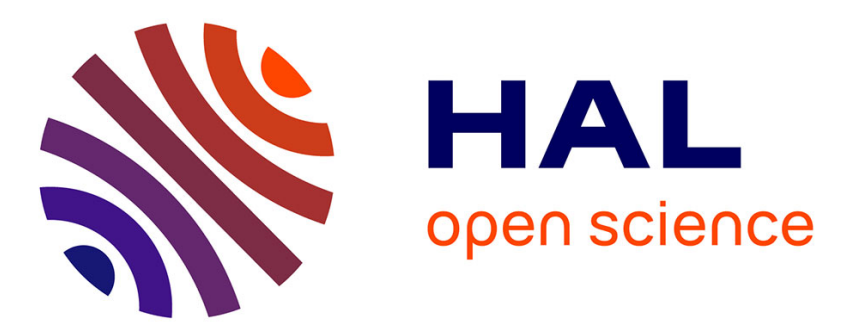

\title{
Silence numérique et injonction de discours : vers un discours socio-affectif-cognitif intégratif en pratique d'une langue étrangère
}

Joséphine Rémon

\section{- To cite this version:}

Joséphine Rémon. Silence numérique et injonction de discours : vers un discours socio-affectif-cognitif intégratif en pratique d'une langue étrangère. Recherche et pratiques pédagogiques en langues de spécialité - Cahiers de l'APLIUT, 2018, Vol. 37 №2, 10.4000/apliut.6299 . halshs-01938756

\section{HAL Id: halshs-01938756 \\ https://shs.hal.science/halshs-01938756}

Submitted on 28 Nov 2018

HAL is a multi-disciplinary open access archive for the deposit and dissemination of scientific research documents, whether they are published or not. The documents may come from teaching and research institutions in France or abroad, or from public or private research centers.
L'archive ouverte pluridisciplinaire HAL, est destinée au dépôt et à la diffusion de documents scientifiques de niveau recherche, publiés ou non, émanant des établissements d'enseignement et de recherche français ou étrangers, des laboratoires publics ou privés. 
Cahiers de l'Apliut

Vol. 37 N²$^{\circ} \mid 2018$

Corps, voix et langues dans l'enseignement supérieur

\section{Silence numérique et injonction de discours : vers un discours socio-affectif-cognitif intégratif en pratique d'une langue étrangère}

Digital Silence and Discourse Injunction: Towards an Integrative Socio-AffectiveCognitive Discourse in Foreign Language Practice

Joséphine Rémon

\section{OpenEdition}

\section{Journals}

Édition électronique

URL : http://journals.openedition.org/apliut/6299

DOI : 10.4000/apliut.6299

ISSN : 2119-5242

Éditeur

APLIUT

Ce document vous est offert par Université Lumière Lyon 2

UNR-RA

Référence électronique

Joséphine Rémon, «Silence numérique et injonction de discours : vers un discours socio-affectifcognitif intégratif en pratique d'une langue étrangère ", Recherche et pratiques pédagogiques en langues de spécialité [En ligne], Vol. 37 N² | 2018, mis en ligne le 30 octobre 2018, consulté le 28 novembre 2018. URL : http://journals.openedition.org/apliut/6299; DOI : 10.4000/apliut.6299

Ce document a été généré automatiquement le 28 novembre 2018.

Association des Professeurs de Langues des Instituts Universitaires de Technologie 


\section{Silence numérique et injonction de discours : vers un discours socio- affectif-cognitif intégratif en pratique d'une langue étrangère}

Digital Silence and Discourse Injunction: Towards an Integrative Socio-AffectiveCognitive Discourse in Foreign Language Practice

Joséphine Rémon

\section{Introduction}

1 Remettre le corps et la voix au centre du dispositif d'enseignement / apprentissage, c'est aussi donner toute sa place au silence, surtout dans un contexte numérique. « Il faut [...] comprendre ce silence numérique dans une acception large du mot silence, dans la mesure où ce qui est ainsi désigné n'est pas l'interruption de la parole naturelle " (Théviot 9). Caranfa (218) suppose que silence, discours et connaissance de soi s'impliquent l'un l'autre et propose de passer d'un mode d'apprentissage par le discours (la connaissance) à un mode d'apprentissage par le silence (la non-connaissance) ${ }^{1}$ (Caranfa 227). C'est par cette entrée, le «silence » ou la non-expression, que nous nous proposons de contribuer à une réflexion sur voix et langues dans l'enseignement supérieur. À travers l'analyse d'un corpus de contributions écrites d'étudiants, nous mettons en lumière les traces de l'injonction de discours et ses effets. Nos unités d'analyse sont donc des segments de discours qui renvoient à la production de discours ou à l'absence de production de discours, ou qui portent des traces du dépassement de cette dialectique. Notre question de recherche porte sur les effets de la multiplication d'espaces de production langagière sur un forum d'apprentissage à distance. Comment favoriser l'engagement des apprenants en leur donnant la parole, sans pour autant créer artificiellement de la non-participation avec des espaces redondants? Les questions d'abandon en formation à distance ont été traitées de manière extensive dans les 
dernières années. Desmarais étudie la persévérance, tandis que Dussarp étudie l'abandon en lien avec le socio-affectif. Albero (24) pointe le décalage entre potentialités de l'environnement et dispositif vécu par chacun :

On découvre qu'il existe autant de dispositifs vécus que de sujets, chacun actualisant à sa manière les potentialités offertes par l'environnement de formation, selon le sens qu'elles prennent dans son parcours, dans l'ordre de ses priorités et dans ses préoccupations du moment.

2 Notre propos est de proposer une typologie du positionnement socio-affectif par rapport au silence en ligne, à travers la définition de régimes de positionnement par rapport à ce non-discours.

\section{Contexte pédagogique}

Dans le cadre d'un module collaboratif de huit semaines, une quarantaine d'étudiants en master de Sciences de l'éducation de l'université de Lyon pratiquent l'anglais et le numérique conjointement. Dans ce contexte, la production de discours (dans notre cas, la production écrite) est cruciale en raison de plusieurs paramètres: il s'agit d'un enseignement à distance, dans lequel la tâche et la production langagière se confondent. La tâche est de surcroît collaborative. En effet, il est demandé aux étudiants de négocier en anglais sur la plate-forme dédiée pour construire ensemble une école virtuelle en utilisant un logiciel de fiction interactive. Parallèlement, il est demandé aux étudiants d'utiliser l'outil collaboratif seesaw.com, qui permet de rédiger, sous la forme de brèves repérées sur un calendrier, un carnet de bord d'apprentissage, chacun ayant accès aux carnets de ses pairs.

4 En ce sens, l'environnement d'apprentissage mis en place pour ce module adhère à une « pédagogie du développement » telle que définie par Albero (42):

Les pédagogies du développement, qui ont pour objectif l'autostructuration interne du sujet à partir de ses interactions avec l'environnement, recherchent la diversification des espaces et des méthodes de travail et d'exploration, d'aide et de communication. Elles offrent une palette de possibles qui vise à induire une interactivité importante. (Albero 42)

5 Un équilibre reste cependant à trouver, avec une injonction de discours d'autant plus forte que, dans l'environnement en question, l'évaluation, même si elle ne porte pas sur la qualité langagière en soi, n'a d'autre support que les traces écrites laissées par les étudiants sur la plate-forme.

\section{Contexte théorique : silence et injonction de discours}

6 Nous présentons ici les éléments théoriques permettant d'appréhender la notion de silence dans un contexte numérique. Qu'en est-il des «silences", entendus non pas au sens d'absence de vocalisation, mais d'absence d'occupation d'espace de discours? Dans une situation d'enseignement à distance, le silence s'interprète en termes de présence. À cet égard, nous retenons les trois dimensions de la présence en e-learning proposées par Jézégou (18) : cognitive, socio-affective et pédagogique. Selon Jézégou (22), la présence cognitive 
résulte des transactions existantes entre les apprenants pour résoudre de façon conjointe et commune une situation problématique. Les transactions sont des interactions sociales de confrontations de points de vue, d'ajustements mutuels, de négociations et de délibérations qui témoignent d'une collaboration à distance entre les apprenants au sein d'un espace numérique de communication. (Jézégou 22)

Celle-ci est soutenue par la présence socio-affective qui résulte des interactions sociales entre apprenants, et par la présence pédagogique, qui résulte des interactions sociales entre formateur et apprenants. Ainsi, lorsque nous parlons plus loin de tissage socioaffectif-cognitif, nous entendons un discours qui mêle des références à la résolution de la tâche et « des interactions sociales basées sur la symétrie de la relation et sur l'aménité » (Jézégou 9).

8 Cisel propose une typologie de l'attrition en formation à distance, qui va du retrait volontaire à l'échec académique (note insuffisante, date limite dépassée, problèmes de discipline), en passant par la réinscription dans un autre cursus. Il distingue également la non-participation en lien avec une absence d'intention du sujet, ou en lien avec le ciblage qui correspond à l'intention de la réalisation d'un objectif personnel.

9 L'absence de production langagière, quant à elle, qui se distingue de l'abandon, peut avoir de nombreuses causes telles que la non-compréhension des consignes, la mésentente avec un tuteur ou les pairs, des difficultés techniques, des problèmes de santé, un moment de découragement, etc. Dussarp et Paquelin (24) proposent les variables suivantes, générant de la « solitude à distance » et, par là, de la non-participation :

des variables propres au dispositif (dysfonctionnements, faiblesse de la relation enseignant-apprenant) et [...] des variables propres à l'apprenant (crainte de la médiatisation de la communication, oser contacter à distance, craintes d'incompréhensions dues au médium utilisé, « résistance » face à l'outil, etc.). (Dussarp et Paquelin 24)

10 Audran et Garcin (11) étudient « les relations qui peuvent exister entre certaines formes de participation dans les forums et des indicateurs d'apprentissage ». Ils rappellent que les données de connexion sont un indicateur imprécis, tandis que les messages postés sont un indicateur de participation "active", sans que le silence soit pour autant nécessairement lié à la passivité (Audran et Garcin 44). Ces auteurs pointent notamment le « poids de l'activité de veille, ou de 'lecture seule' dans la réussite » (Audran et Garcin 44).

11 Notre étude ne prend pas cependant pour objet principal les effets du silence ou ses causes, mais plutôt un positionnement socio-affectif par rapport à la difficulté ou à l'aisance à répondre à des injonctions à produire un discours, en langue étrangère, de surcroît. Si l'on reprend les trois niveaux de confiance présentés par Eneau (7), confiance institutionnelle, confiance interpersonnelle et confiance personnelle, on voit que notre problématique interpelle à la fois ces trois niveaux. En effet, la confiance dans le dispositif peut être minée si la multiplication des espaces de production langagière n'est pas perçue comme ayant du sens. En outre, la confiance interpersonnelle peut être remise en question si certains apprenants seulement parviennent à s'emparer d'un espace. Et, enfin, la confiance personnelle peut être atteinte par l'impression que l'on ne parvient pas à répondre aux exigences perçues. Albero et Kaiser (14) affirment que « la FOAD [formation ouverte et à distance] s'avère être une modalité inadéquate pour les personnes caractérisées par une attitude sceptique vis-à-vis de leur capacité d'apprentissage ». Albero et Kaiser (6) indiquent que « la désorientation cognitive peut venir renforcer, au 
plan émotionnel, un sentiment d'isolement ». Ce risque de désorientation cognitive est réel avec la multiplication des espaces et, avec elle, la multiplication des injonctions à produire du langage, et le risque de créer artificiellement des traces de non-participation.

\section{Visualisation quantitative}

12 Notre étude, avant tout qualitative, prend appui sur une prégnance du silence numérique telle qu'elle apparait sur les graphes présentés ci-dessous. Nous exposons d'abord notre choix de visualisation, et la problématique de la création artificielle de traces de nonparticipation. Les graphes ont été générés à partir des fichiers Excel de données quantitatives disponibles pour avril et mai 2017 sur le forum (nombre de messages par étudiant et par date) et sur l'application de carnet de bord d'apprentissage, qui associe un nombre de messages à un utilisateur, et un message à une date du calendrier.

\subsection{Visualiser le non-discours : graphes de répartition temporelle}

13 La structure d'un forum ne permet pas toujours de visualiser facilement les non-discours, même si des outils de suivi existent de nos jours sur les plates-formes dédiées. De nombreux types de représentations des données d'un forum existent (Dimitracopoulou et Bruillard ; Teutsch et al. ; Bruillard et Huynh Kim ; Holmer ; Herring) et ont été discutés. Audran et Garcin (38) pointent le besoin d'une visualisation temporelle pour :

identifier les différentes phases de participation tant pour les « posteurs » que pour les «zyeuteurs", comme, par exemple, le temps ou le nombre de connexions, les périodes laissant apparaître un accroissement ou un amoindrissement de la participation. (Audran et Garcin 38)

Dans le même ordre d'idées, nous retenons une représentation en lien avec la notion de chronotope, empruntée à Grassin et Lemke. Grassin (8) en donne la définition suivante : «Un chronotope est un ensemble d'activités dont le sens social est défini collectivement à la fois par le lieu et par le temps où elles se déroulent ». Grassin analyse les traces d'activité «selon la modalité sémiotique de la contribution » (26) et génère des graphes de répartition temporelle. Nous avons généré un graphe similaire, tridimensionnel, à partir des données quantitatives recueillies automatiquement sur la plate-forme d'apprentissage. Ainsi, ici durant le mois d'avril, en fonction de la date de publication et de l'identité de l'étudiant, s'affichent les contributions sous forme de courbe. En creux se dessinent alors tous les espaces de non-discours (figure 1). 


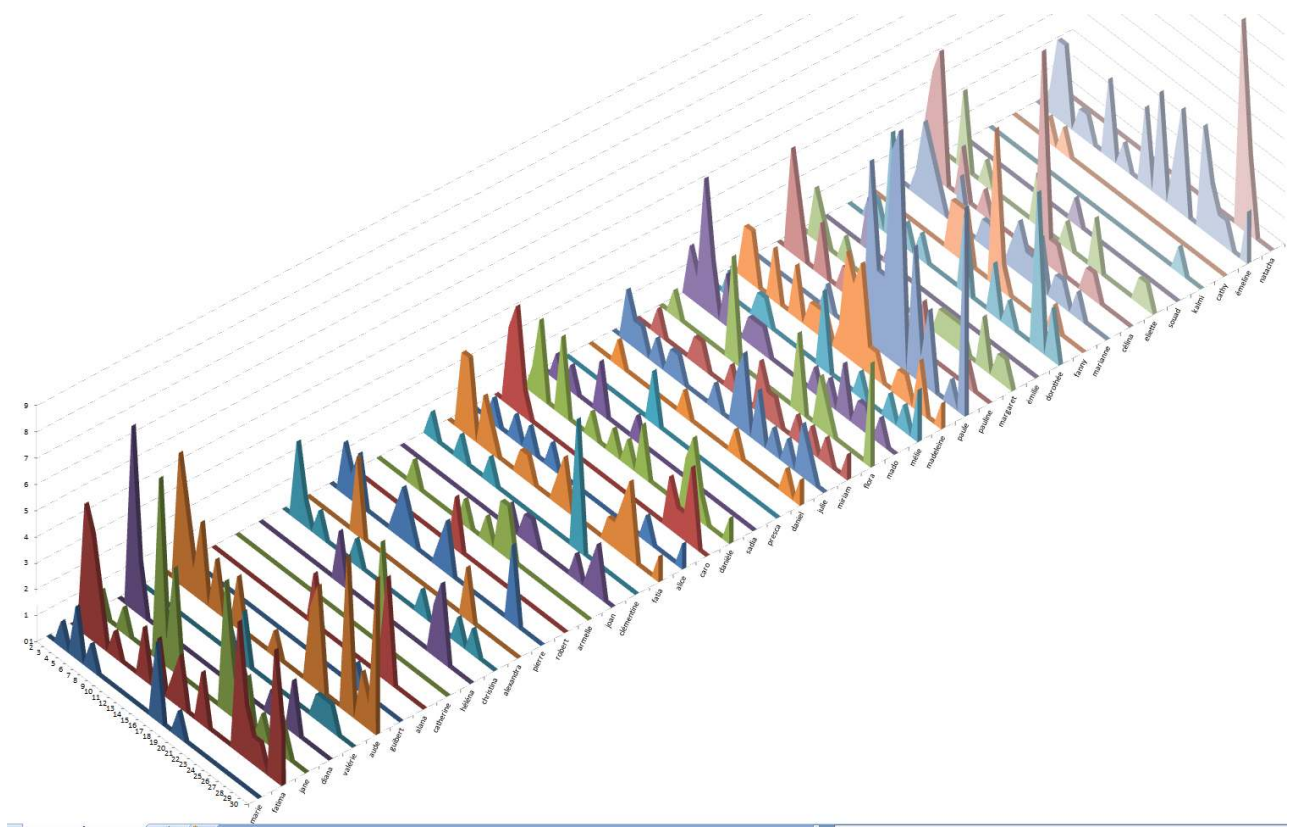

Représentation tridimensionnelle à partir des données quantitatives recueillies automatiquement sur la plate-forme d'apprentissage, générée via le logiciel Excel.

15 L'axe vertical indique le nombre de messages, l'ordonnée affiche le nom des étudiants, et l'abscisse, les 30 jours successifs pendant lesquels les apprenants ont posté leurs messages. Ainsi, on voit par exemple, pour l'étudiante qui a la plus grande zone de couleur, Pauline ${ }^{2}$, qu'elle a été particulièrement active vers le milieu du mois, tandis qu'Alana n'a pratiquement pas contribué.

Comme nous l'avons évoqué ci-dessus, un élément vient ajouter de la complexité au dispositif. En effet, en plus de la négociation sur le forum en vue de la construction d'une école virtuelle en sous-groupe, il est demandé aux étudiants de consigner sur un carnet de bord d'apprentissage les événements d'apprentissage qu'ils jugent dignes d'intérêt. Cette consigne génère 411 items publiés sur le carnet de bord électronique seesaw.com. Sur le graphe suivant (figure 2), généré là aussi à partir des données quantitatives recueillies automatiquement par la plate-forme d'apprentissage, sur une période d'un mois, on affiche, pour chaque étudiant, à la fois les contributions sur le carnet de bord (en rouge) et les contributions sur le forum (en bleu). Les axes sont les mêmes que pour la figure 1, c'est-à-dire noms des étudiants, jour du mois et nombre de messages à la verticale. 
Figure 2 : Les contributions sur le forum et les contributions sur le carnet de bord, par étudiant

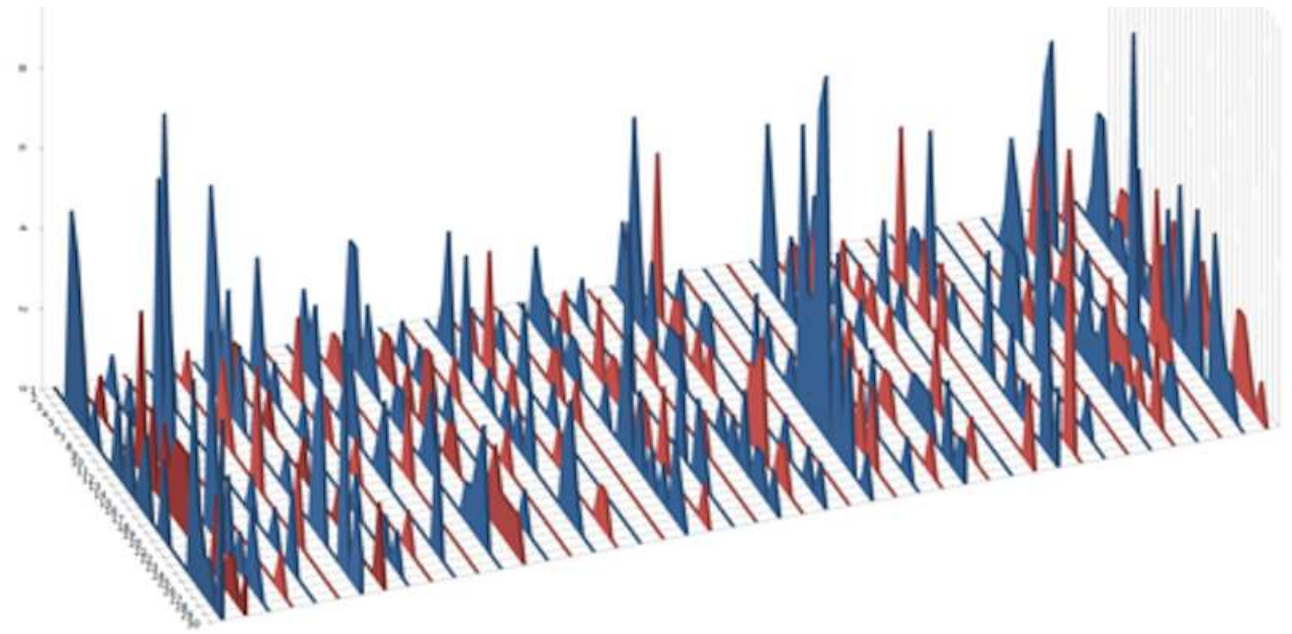

Représentation tridimensionnelle à partir des données quantitatives recueillies automatiquement sur la plate-forme d'apprentissage

Ainsi, on crée une trace qui met en évidence l'utilisation moins intensive du carnet de bord par rapport au forum, ce qui est en accord avec les consignes puisque ces dernières donnent la plate-forme d'apprentissage pour principal lieu de négociation entre pairs. Ce non-discours relatif sur le carnet de bord est une création dans une certaine mesure artificielle résultant de la multiplication des espaces et des injonctions.

Si l'on prend pour exemple la visualisation pour un étudiant parmi les plus productifs (figure 3), des zones de « silence » apparaissent également. 
Figure 3 : Activité sur le forum et sur le carnet de bord pour un étudiant spécifique, parmi les plus productifs

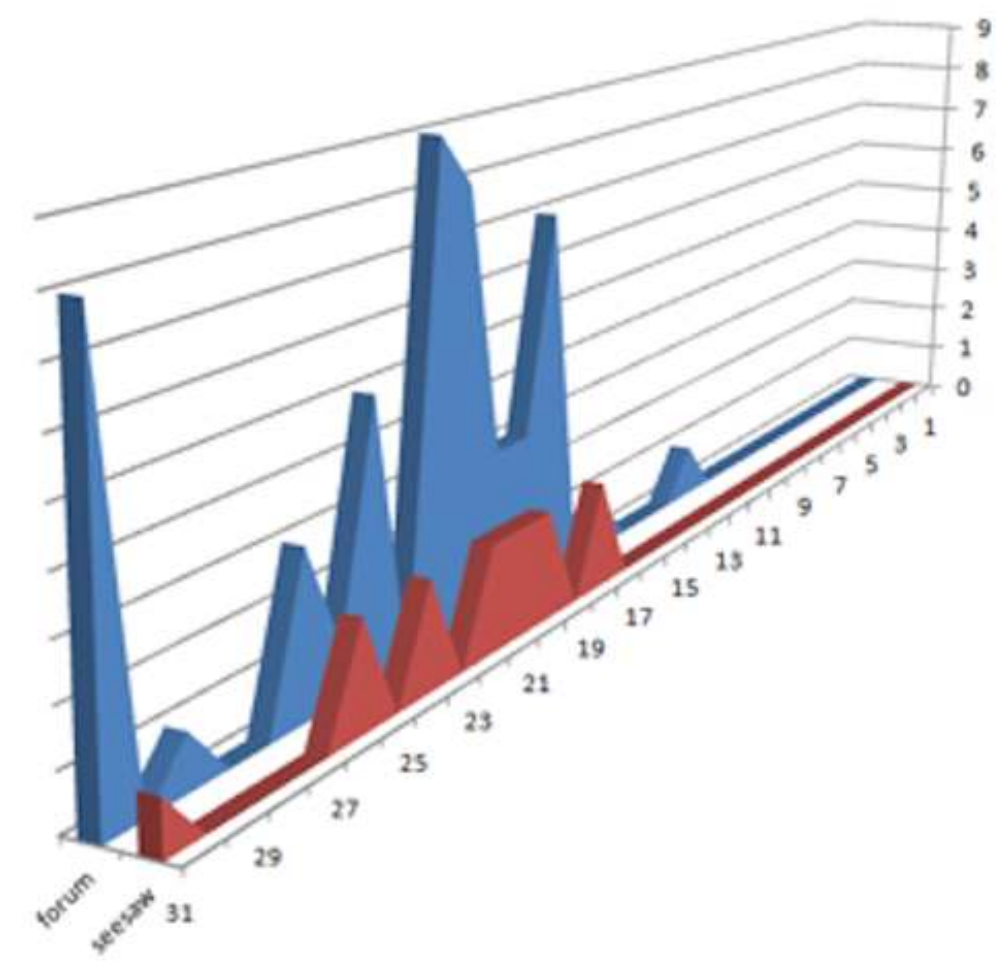

Représentation tridimensionnelle à partir des données quantitatives recueillies automatiquement sur la plate-forme d'apprentissage

Ici, l'axe vertical indique le nombre de messages, tandis que l'axe horizontal indique les jours du mois. La plus grande zone de couleur correspond au forum, tandis que la zone plus restreinte correspond aux publications sur le carnet de bord seesaw.com. Cette même visualisation des non-discours apparait par un autre biais, celui de l'outil calendrier du carnet de bord. En effet, seesaw.com permet d'accéder aux publications des étudiants par date, disposées dans un calendrier. Ainsi, si l'on prend l'exemple du mois de mai 2017, il y a un pic d'activité certains jours, par exemple le jeudi 4 mai ou le mardi 23 mai, avec en creux des zones de relative inactivité (figure 4). 
Figure 4 : Activité sur le forum et sur le carnet de bord pour un étudiant spécifique, parmi les plus productifs

\begin{tabular}{|c|c|c|c|c|c|c|}
\hline \multicolumn{7}{|c|}{ May 2017} \\
\hline Sunday & Monday & Tuesday & Wednesday & Thursday & Friday & Saturday \\
\hline & 1 & 2 & 3 & & 5 & 6 \\
\hline & 4 items & 10 items & 6 items & 15 items & 6 items & 5 items \\
\hline 7 & 8 & 9 & 10 & 11 & 12 & 13 \\
\hline 3 items & 6 items & 6 items & 5 items & 14 items & 4 items & 1 item \\
\hline 14 & 15 & 16 & 17 & 18 & 19 & 20 \\
\hline 7 items & 9 items & 8 items & 1 item & 10 items & 5 items & 9 items \\
\hline 21 & 22 & 23 & 24 & 25 & 26 & 27 \\
\hline 4 items & 12 items & 19 items & 9 items & 1 item & 1 item & 10 items \\
\hline 28 & 29 & 30 & 31 & & & \\
\hline 6 items & 1 item & 1 item & & & & \\
\hline
\end{tabular}

Calendrier issu de la plate-forme seesaw.com

cartes conceptuelles et des robots conversationnels, en testant les outils numériques correspondants, et cette tâche occasionne traditionnellement un regain d'activité.

\subsection{Créer des traces en creux}

Entrer dans une réflexion sur la voix dans la pratique des langues dans l'enseignement supérieur, et, par là, sur le silence, nous amène, surtout dans un contexte numérique, à interroger la trace ou l'absence de traces, et la visualisation de celles-ci. Comme l'indique Bonenfant (17), « [d]ans le contexte de la communication, il y a production intentionnelle de signes, mais ces signes portent en eux-mêmes plus que leur signification : ils portent également des traces de leur production ». Dans un contexte numérique avec recueil automatique de données quantitatives, les discours comme les non-discours sont réifiés : « la trace marque une absence dans le présent » (Bonenfant 20).

Dès lors que l'on s'intéresse aux traces de non-participation, on peut se demander comment opérationnaliser la collecte des données pour observer de manière qualitative ce que les graphes montrent du point de vue quantitatif. En effet, Audran et Garcin (37) précisent que :

[l']interprétation des données peut difficilement reposer uniquement sur une question de quantité ou de proximité, alors que la nature des messages échangés joue d'évidence un grand rôle, certains d'entre eux étant majoritairement informatifs (de type questions et réponses), et d'autres plus conversationnels. Le choix qui a été fait ici étant de se centrer sur les aspects quantitatifs, des recherches seront donc à mener sur les aspects qualitatifs. Audran et Garcin (37)

Il ne s'agit pas dans notre cas de collecter les silences, mais de collecter les discours de positionnement sur l'injonction à produire du langage. Comme indiqué en introduction, notre analyse porte donc sur des segments de discours qui renvoient à la production de 
discours ou à l'absence de production de discours, ou qui portent des traces du dépassement de cette dialectique.

Ainsi, en multipliant les espaces, on multiplie les possibilités de non-discours. On court le risque de mettre en difficulté des étudiants en créant « en creux » une trace non assumée, un « silence » sur le plan individuel et sur le plan du cours. Avec cette absence de discours qui laisse une trace en creux, l'injonction pédagogique de participation se fait plus que jamais sentir sous forme d'injonction de discours ou d'injonction de justification de nondiscours. Tout "silence " amène à justifier ce même silence. Nous étudions plusieurs occurrences de ce phénomène apparues dans notre corpus.

\section{4. Étude qualitative}

À travers une étude qualitative du corpus, nous mettons en évidence que, au-delà d'être la trace de difficultés, le silence numérique s'inscrit dans des régimes de positionnement par rapport à l'injonction de discours, ou au-delà de cette injonction en donnant corps à un discours socio-affectif-cognitif. Ce tissage peut être défini comme la présence simultanée dans un même message d'éléments relevant du socio-affectif et du cognitif. Nous entendons régime au sens de Teil (28): «une organisation appuyée sur des équipements, instruments, procédures, raisonnements qui orientent l'action collective tout en atténuant l'idée trop forte d'action organisée ». Nous avons procédé par lecture flottante puis par saturation des catégories, selon la méthode détaillée par Lejeune ${ }^{3}$.

\subsection{Le non-discours, trace de difficultés}

Le «silence» dans un contexte numérique peut être classiquement un signe de difficultés, et les discours font alors mention de celles-ci. Ainsi, dans l'exemple ci-dessous, l'étudiante, qui ne publie rien sur le forum entre le 5 et le 25 avril, lance un appel à l'aide le 25 («Help! :-) :-) ») en utilisant le verbe « help » suivi de deux émoticônes et d'un point d'exclamation.

Message : RE : a cow's dream

C. 25 avril 2017 17:59

:-) :-) With my TER and our institution , i dream about prison in English and in french!!! Help! :-) :-)

Si les émoticônes semblent relativiser l'urgence de son appel à l'aide le 25 avril, elle renouvelle celui-ci le 27 avril. Cette fois, c'est un appel à l'aide en français, dans une rubrique dédiée appelée "En cas d'urgence ", dans laquelle les étudiants ont le droit de publier en français. Il est très rare que les étudiants utilisent cette rubrique. De la même manière, son silence sur le carnet de bord lui pose également problème. Elle le mentionne le 22 mai sur le forum en indiquant qu'elle essaye de rattraper son retard ( just trying to get caught up ») en commençant à publier sur seesaw.com. Elle publie dans la foulée huit messages à cette date sur le carnet de bord.

\subsection{Entre discours et non-discours}

Dans ce contexte de relative urgence à produire du discours tel qu'il est ressenti par certains étudiants, de nombreuses contributions relèvent de la tension entre discours et 
non-discours, selon des modalités diverses. Le seul discours produit est parfois la justification même du non-discours, comme dans le premier cas de figure présenté ici en 5.2.1. Nous voyons d'autres régimes de production ou de non-production de discours plus bas, à savoir le régime du refus du non-discours, le régime du déni du non-discours, le régime de l'anticipation du non-discours.

\subsubsection{Régime de la justification du non-discours}

Ainsi, une étudiante, qui abandonne par la suite le module d'anglais, publie sur le forum les deux uniques messages que nous reproduisons ci-dessous, chacun mentionnant les difficultés rencontrées (la naissance d'un enfant et le concours d'enseignant) et l'impossibilité de participer. Elle n'utilise pas le carnet de bord.

Thème : Time Space School 13 avril 2017 14:04

Hello everyone, I have meet some difficulties to connect me on the platform but now everything is ok.

Thème : Week 6 : bots ! 10 mai 2017 09:30

Hello Miss R., Hello everyone,

Please excuse my delay for this work. I passed my CRPE in April and at the same time I await the arrival of my second child very soon. It's a little difficult to reconcile everything.

Dans cet autre exemple, l'outil calendrier du carnet de bord d'apprentissage numérique permet d'identifier une période d'inactivité de quelques jours, entre le 20 et le 23 avril. On trouve la justification de ce non-discours dans un message sur le forum (ci-dessous), repris à l'identique sur le carnet de bord à la même date (figure 5). En effet, l'étudiante indique avoir été malade (« a very sick week »).

Thème : WEEK 3 (already !!) F. 24 avril 2017 12:26

Hello Mrs R.

I hope you are well! After a very sick week, i recover gently and i am back to work. Today i test the games in lines. It amuses me well! Here is my first comix, i love it. [...] 


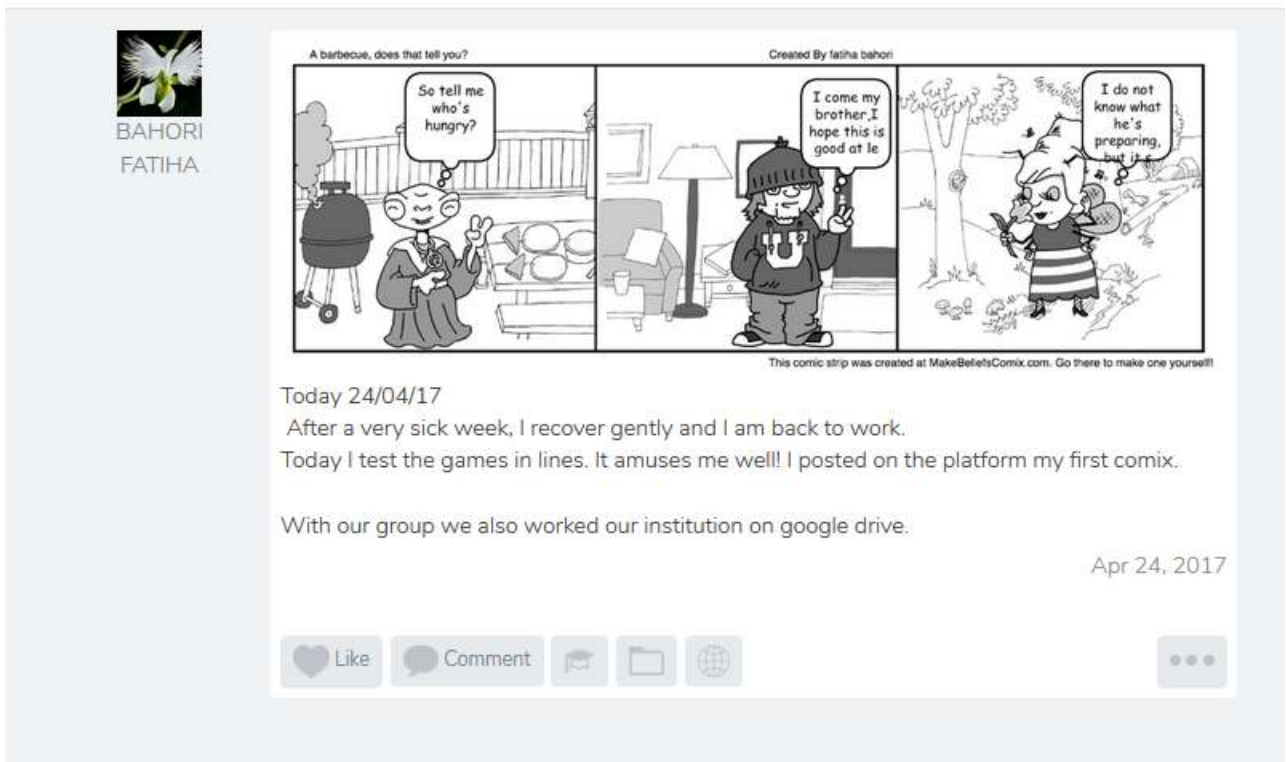

Extrait du carnet de bord montrant une bande dessinée créée par l'étudiante à l'aide de l'outil numérique makebeliefscomix.com

Dans certains cas, la justification du non-discours masque un tissage socio-affectif, comme dans l'exemple que nous décrivons maintenant. Un autre étudiant rencontre des difficultés diverses, et sur les quatre messages qu'il a réussi à publier sur le forum, l'un est un message d'excuse pour son relatif «silence» qui se traduit en termes d'absence ( $I$ also apologize for my absence, I'm present now »).

24 avril

Hello Mrs. R.,

I'm ready to participate. I've registered on the website.

I also apologize for my absence, I'm present now and I will participate to all next activities

Best regards,

Paradoxalement, par l'intermédiaire de la publication d'une carte conceptuelle, intitulée « Ma vie en 2017 » (figure 6), créée grâce à un outil numérique qu'il a testé, il explique avec beaucoup de détails, sur un ton humoristique, sa situation au moment du module d'anglais, en hiérarchisant ses problèmes: "Pas assez de place à la maison pour travailler ", «Je mange trop », « Ma mère », « Ma compagne ", « Ma fille de 10 mois », etc. Ainsi, le non-discours apparent masque une expression détaillée d'une intimité livrée en confiance, et qui contribue au climat socio-affectif de l'ensemble du module. 


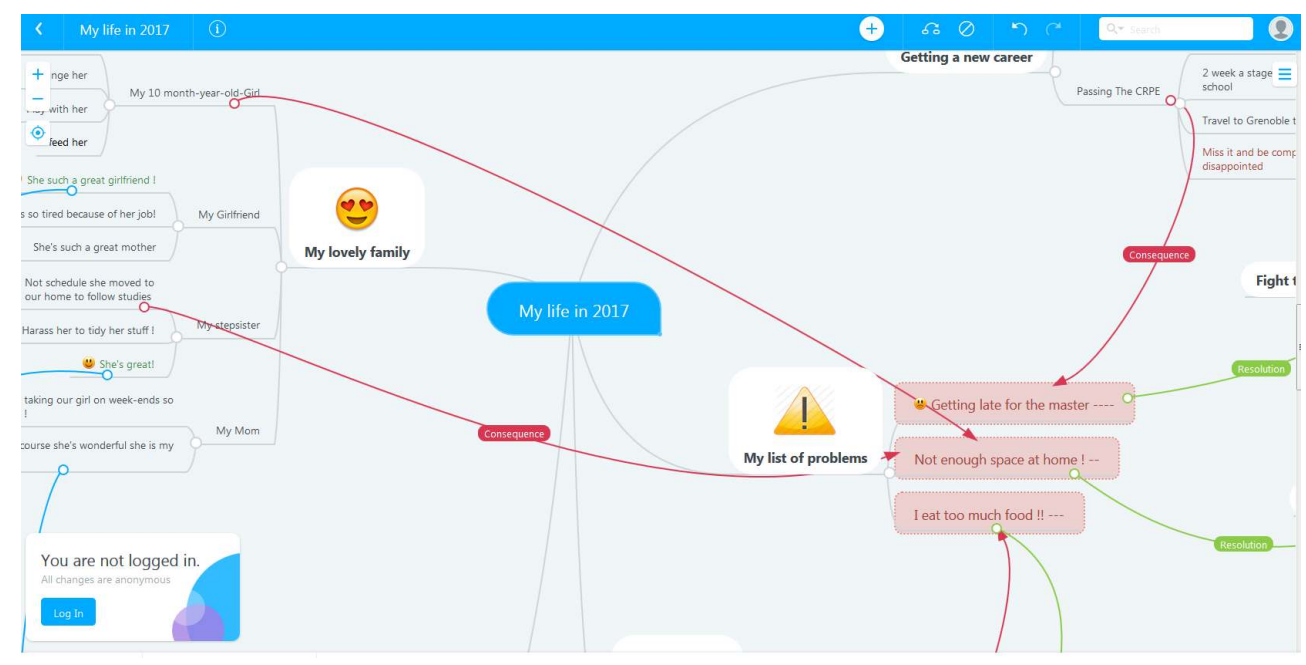

Carte conceptuelle créée par un étudiant à l'aide de l'outil numérique MindMeister

Dans d'autres exemples, la justification du non-discours trouve un écho dans le discours des pairs. En effet, un autre cas de figure, à regarder à travers le prisme discours/nondiscours, est le cas de cette étudiante qui, elle aussi, publie très peu de messages sur le forum et rien sur le carnet de bord, mais dont la présence socio-affective est étoffée par les commentaires de ses pairs, en réponse à un message qu'elle a posté sur l'école virtuelle en construction.

Elle rencontre des difficultés, comme elle l'indique le 21 avril 2017 :

Good Evening Mrs R.,

I had a lot of trouble with my work and my computeur, so I catch up now.

Mais, parallèlement, en effet, ses pairs donnent un certain écho au peu de commentaires qu'elle écrit dans le sous-groupe de travail :

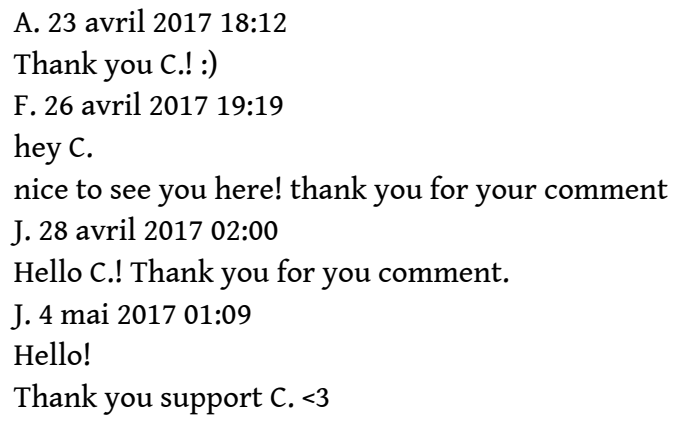

Cette étudiante est finalement autant présente dans les messages de ses pairs que par ses propres publications. Là encore, le «silence » relatif masque une ébauche de tissage socio-affectif.

\subsubsection{Régime du refus du non-discours ou le « refus de se laisser réduire au silence $"$}

Face à l'injonction de discours et au spectre du «silence » que les étudiants se sentent obligés de justifier, le cas suivant est éclairant, car l'étudiante continue à produire du 
discours alors même qu'il lui est signifié qu'elle ne peut plus suivre le module d'anglais. Ainsi, ce refus de se laisser « réduire au silence » signale en creux l'enjeu que représente la réalisation du discours. C'est une étudiante qui n'était pas présente au regroupement initial, comme le montre le message ci-dessous :

K. 6 avril 2017 [...] It's a little difficult for me et some of others students like me cause we couldn't be there in january for grouping. [...]

Elle initie le travail dans un message le 16 avril à destination de la tutrice où elle signale que tout le groupe est « en retard» sur le calendrier (« we are late sorry ») :

H. 16 avril 2017 21:14

Hello Mrs R.,

Thank you for your welcome. We are a group of 4 students and we are late sorry. [...] H.S., K. S., C. S., K.

Et le 27 avril à destination de son groupe :

K. 27 avril 2017 21:59

Hello to my group!

I found websites to help us create the best green shcool. [...] what you think?

Les difficultés rencontrées affleurent le 9 mai lorsque, dans deux messages différents sur le forum, l'étudiante signale qu'elle n'a pas de groupe et qu'elle manque de temps à cause de son travail :

9 mai 2017 [...] I have a problem I have not a group and I begin to work now cause no time in my professionnal life [...]

9 mai 2017 I am very in late for professionnal reason. I have not a group cause it was complicated . [...] Give me your opinion if it's a big problem [...]

La tutrice lui signale dans deux messages successifs qu'elle ne peut pas travailler seule et que la responsable du master lui demande de se présenter à la session de rattrapage pour ce module :

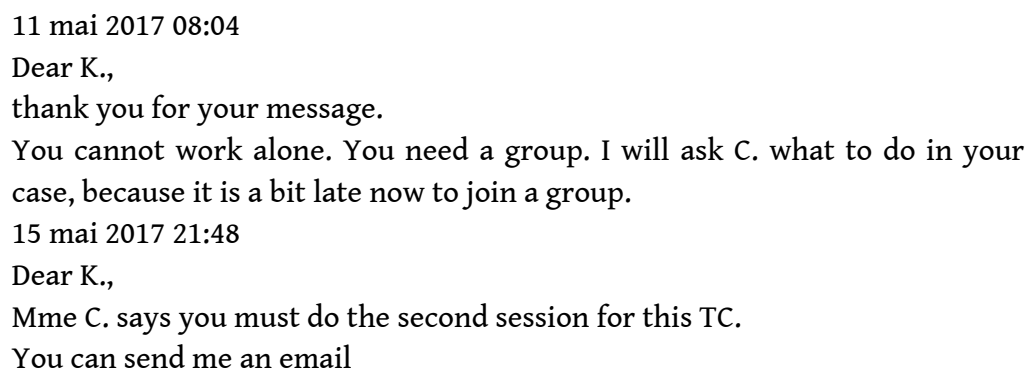

L'étudiante continue de produire du discours régulièrement sur le forum après cela (11 mai, 12 mai, 16 mai, 18 mai avec deux messages, 19 mai, 23 mai avec deux messages, 25 mai et 26 mai). Sur le carnet de bord numérique, elle publie également des messages, qui apparaissent sur le calendrier en date du 11 mai ( 3 items), du 15 mai (2 items), du 18 mai (3 items) et du 23 mai (3 items).

Le 18 mai, il semble affleurer malgré tout une trace de la tension entre injonction de discours et risque d'être réduite au non-discours. En effet, dans la note ci-dessous (figure 7), l'étudiante publie en français, et précise « parce que là j'ai plus les mots ». 
Figure 7. Trace de tension entre injonction de discours et risque de réduction au non-discours

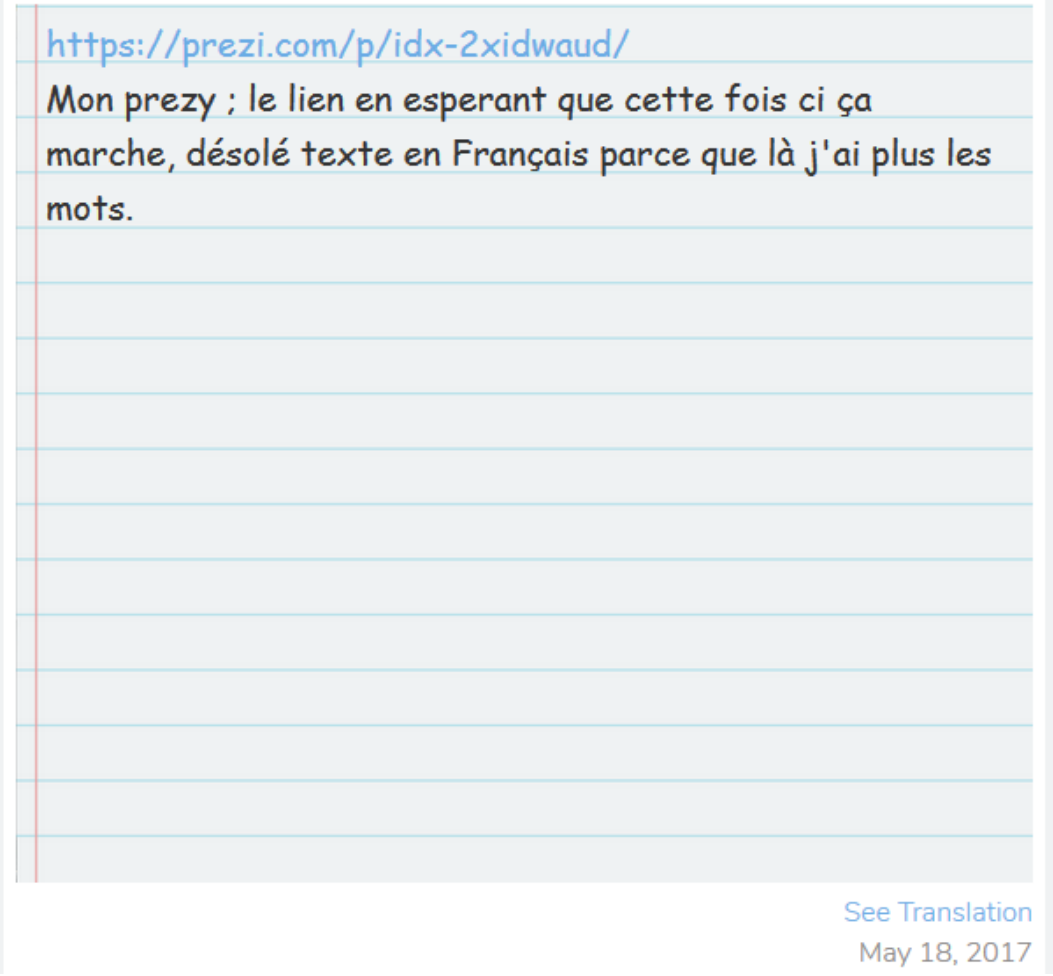

Extrait du carnet de bord rédigé sur l'application seesaw.com

41 Se combine à ce commentaire à caractère affectif une mauvaise manipulation numérique qui lui fait publier une première version d'un diaporama qui est lui-même par inadvertance vide de texte. Ainsi, face à l'injonction de discours, ce régime de positionnement passe par un attachement à produire ce discours, alors même qu'il a été signifié à la participante qu'elle relève désormais d'une autre modalité d'évaluation de son cursus.

\subsubsection{Régime du déni du non-discours : «Silence, quel silence ? »}

42 Les pairs de cette étudiante, membres de son sous-groupe de travail qui a mal fonctionné, ne font cette fois aucune allusion à leur non-discours et publient, en toute fin de module, le 28 mai, après un "silence" complet, une partie des productions demandées. L'une poste même à cette date un message au nom du groupe en proposant de tester l'école virtuelle créée en réponse à la tâche proposée par la tutrice :

Hello everybody,

You can visit our institution !! This is the link : http://[...]

We hope it will work!!!

Thanks

C., H. et K. S.

43 Ce déni à la fois du dysfonctionnement du groupe et du non-discours met là encore en lumière les effets de l'injonction de discours faite dans le cadre du module d'anglais, et le statut du « silence » comme un écueil à éviter. 


\subsubsection{Régime de l'anticipation du non-discours : « Le silence escompté n'aura pas} lieu »

À l'inverse, toujours autour de la tension entre discours et non-discours comme enjeu majeur, une étudiante qui craint de ne pas suffisamment participer en raison de difficultés conjugales donne finalement « corps " à son discours à travers les modes les plus variés, comme nous le voyons ci-après.

En effet, le 10 avril, elle envoie un courrier électronique à la tutrice pour l'informer qu'elle « traverse une période de rupture conjugale ainsi que toutes les complications qui y sont liées, et cela a malheureusement entraîné un peu de retard » dans son travail. Elle publie finalement plus de 100 messages au total, ce qui fait d'elle une des participantes les plus actives.

Sur le carnet de bord, elle fait deux allusions à sa situation, le 17 avril (figure 8) et le 25 avril, et une mention sur le forum ( 30 avril), mais ces mentions mêmes sont l'objet de messages dans lesquels elle mêle socio-affectif et cognitif, répondant par là à la tâche prescrite. Nous en montrons un exemple ci-dessous, dans lequel elle mentionne pêle-mêle "personal difficulties (marital breakdown)», et des éléments concernant l'avancement de ses travaux et de son groupe :

Figure 8 : Mention de difficultés dans un message qui intègre une visée cognitive

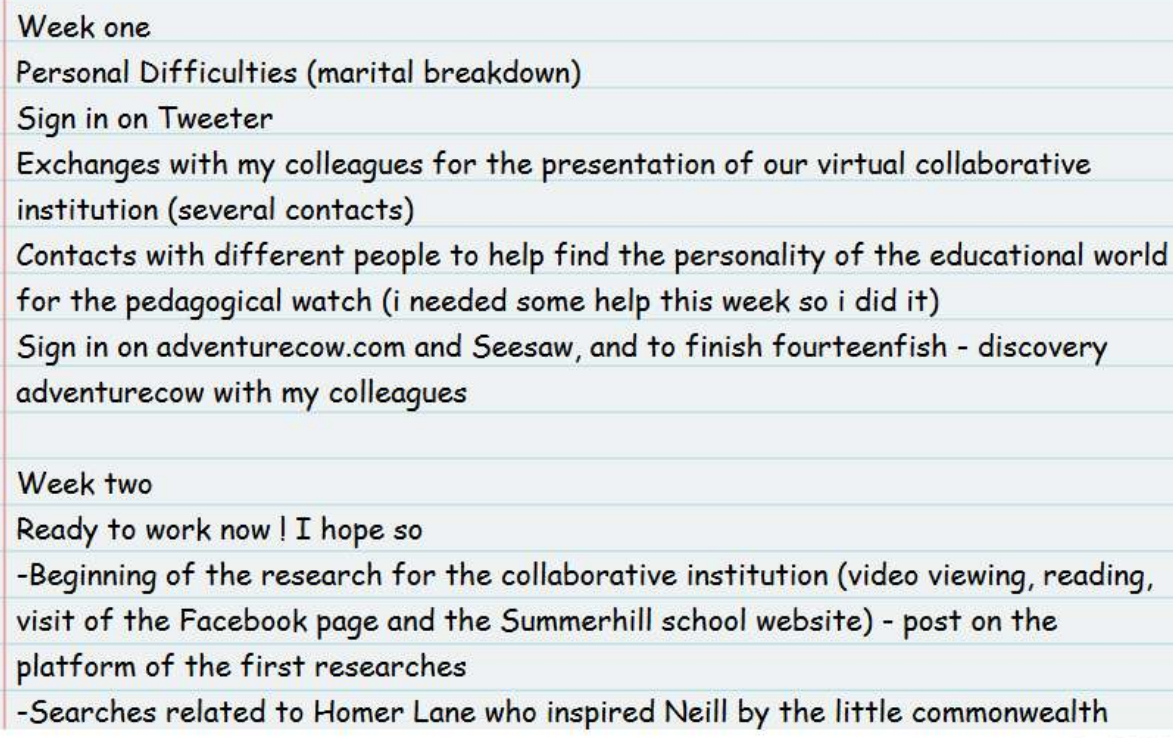

Extrait du carnet de bord rédigé sur l'application seesaw.com

Ainsi, son anxiété concernant son "silence » se traduit au contraire par du discours. Contrairement à sa représentation initiale, elle produit des discours de formes variées. Ainsi, elle publie un dessin réalisé à l'aide d'un outil numérique et une bande dessinée accompagnée d'un fichier son (figures 9 et 10 ci-dessous) : 
Figure 9 : Discours sous la forme d'un dessin créé en ligne

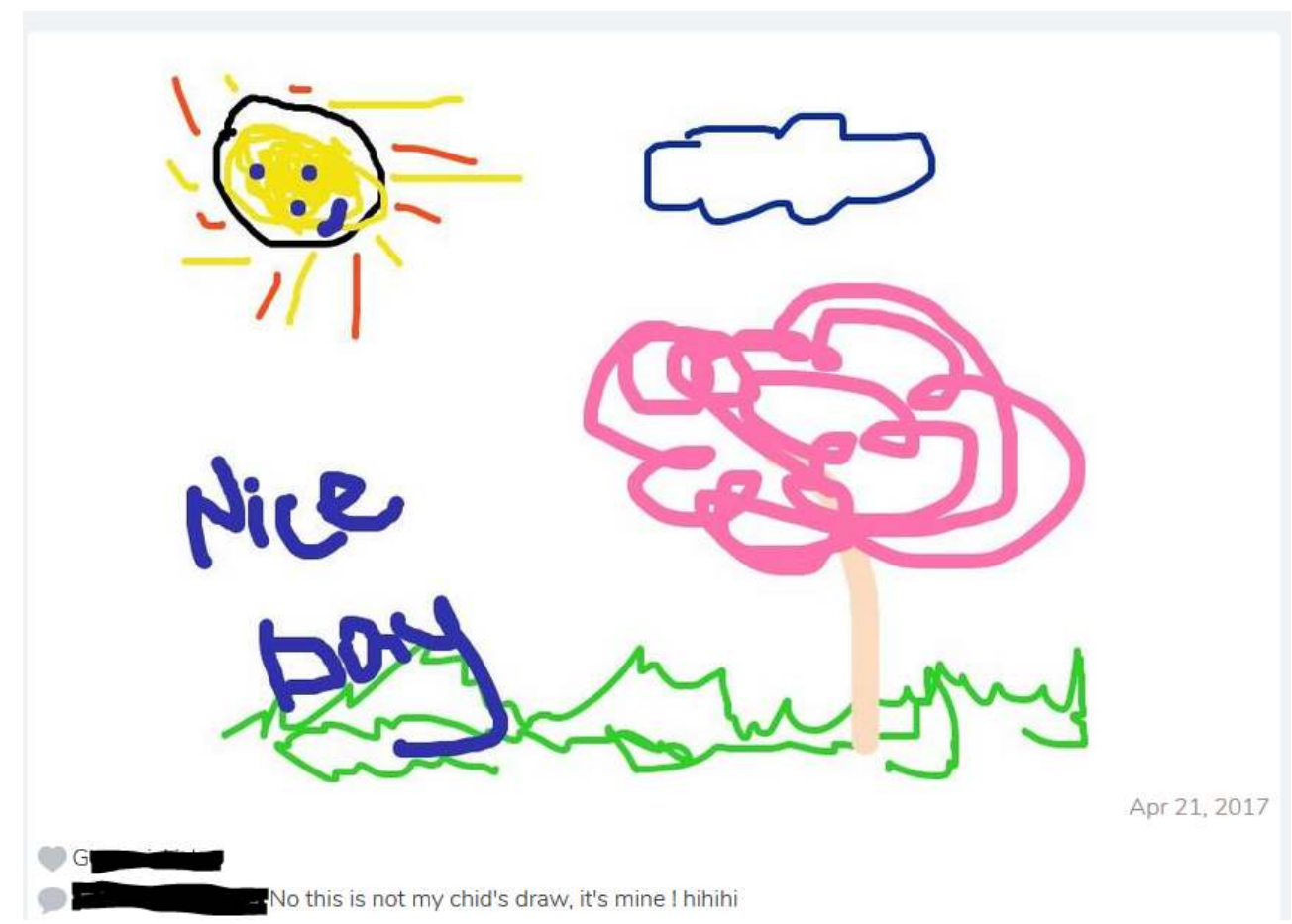

Extrait du carnet de bord rédigé sur l'application seesaw.com, montrant un dessin réalisé par l'étudiante à l'aide d'un outil numérique.

Figure 10 : Bande dessinée accompagnée d'un fichier son

Extrait du carnet de bord rédigé sur l'application seesaw.com, montrant une bande dessinée réalisée par l'étudiante à l'aide de l'outil numérique wwww.makebeliefscomix.com, et incluant un fichier son.

$\mathrm{Ou}$ encore un message à coloration socio-affective (avec la présence de points d'exclamation et d'éléments de lexique tels que «mood», «better », « well », « enjoy », « nice », « good ») accompagné d'un slogan en image (figure 11) : 
Figure 11 : Un message sur le forum accompagné d'un slogan en image

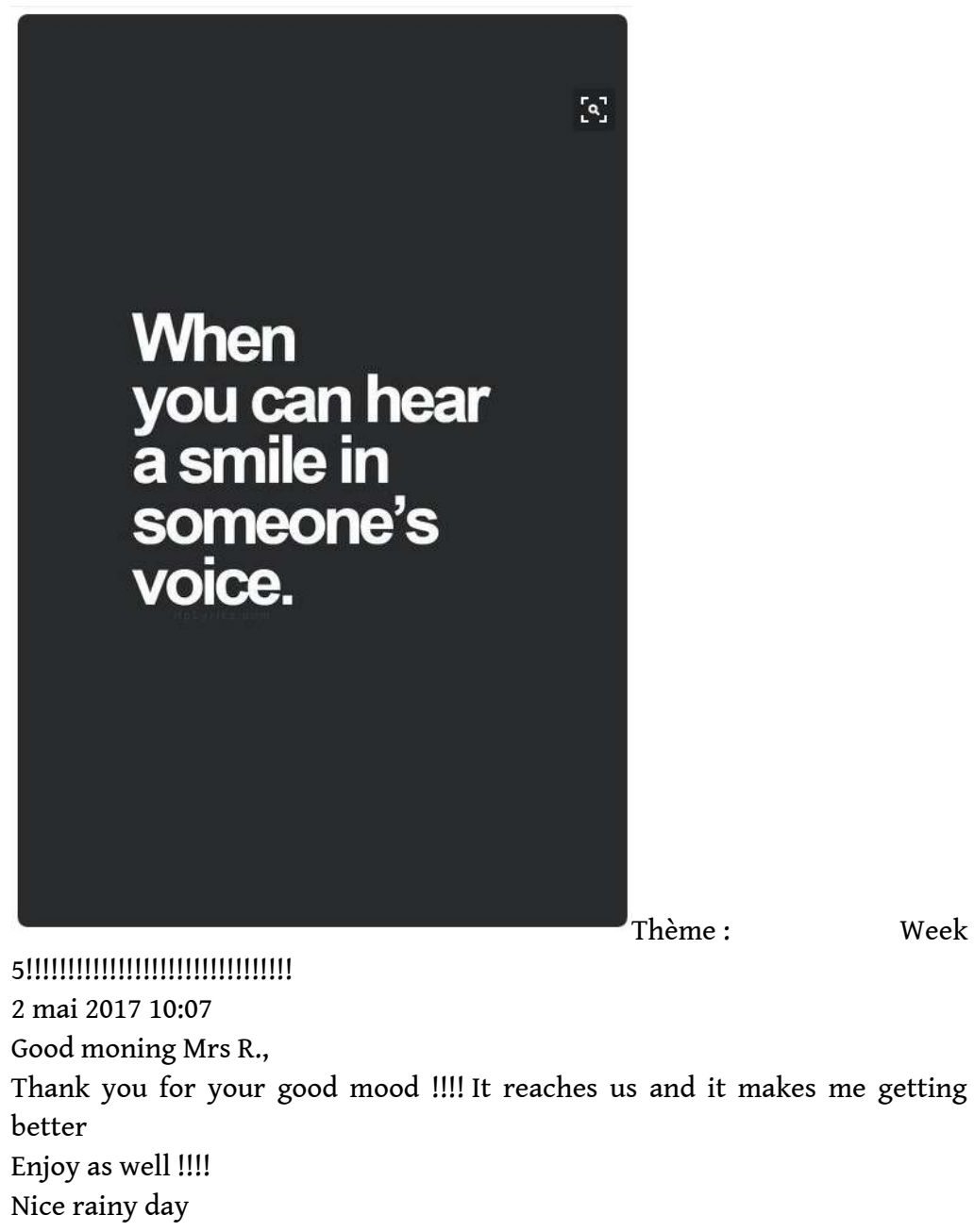

Extrait du forum montrant le message de l'étudiante et une image récupérée sur Internet, puis insérée dans le message.

Ainsi, de nouveau dans la tension entre discours et non-discours, on constate que cette étudiante qui semble capable d'utiliser tout l'éventail des modes de discours à sa disposition éprouve tout de même le besoin initial de se positionner par rapport à l'injonction de discours ressentie, comme on l'a vu ci-dessus avec son message électronique du 10 avril à la tutrice et trois allusions à sa situation personnelle, facteur de risque d'abandon.

\subsection{Au-delà de l'injonction de discours}

Certains étudiants arrivent à dépasser cette injonction de discours pour en faire une contrainte créative. Ils rendent non pertinents les régimes de positionnement au discours/non-discours et parviennent à "donner corps » à du socio-affectif-cognitif en « donnant une voix » à leur famille ou à leurs pairs, en lien avec les tâches pédagogiques.

51 Les références à la famille sont en effet nombreuses. Nous en montrons un exemple cidessous avec une étudiante mentionnant son séjour en Espagne en famille («i was in my 
family in Spain »), et le fait qu'elle a montré à son fils comment créer une tomate animée («I 've shown him that my animated tomato ») :

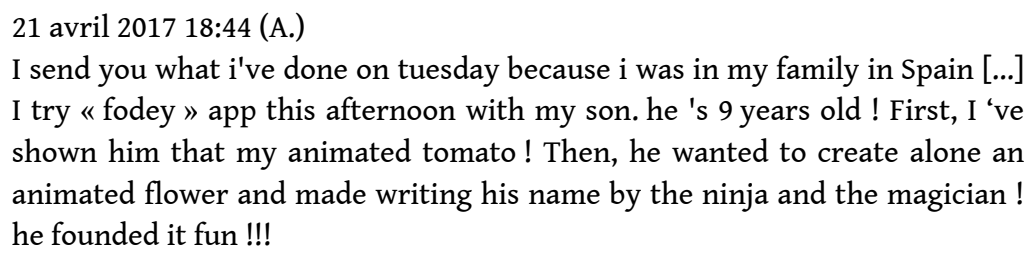

Il est intéressant de noter que le socio-affectif se mêle au cognitif, puisque la mention de la sphère intime à travers la référence aux proches se fait dans le contexte d'un commentaire sur les tâches à effectuer dans le cadre du module d'anglais.

Dans d'autres occurrences, toutefois, non seulement le focus n'est plus sur l'injonction de discours ou sur la justification du non-discours avéré ou présumé, mais sur du socioaffectif, sans même mention du cognitif. Ainsi, une étudiante publie sur le carnet de bord d'apprentissage un dessin humoristique qui représente un enfant dormant en travers du lit de ses parents (figure 12), avec le commentaire : " My life at the moment with a baby boy who doesn't like to sleep !!! ». On pourrait y voir toutefois un lien indirect avec l'injonction de discours et le cognitif, au sens où le manque de sommeil expliquerait des retards éventuels dans le rendu des travaux ou dans la publication de messages.

Figure 12. Dessin humoristique

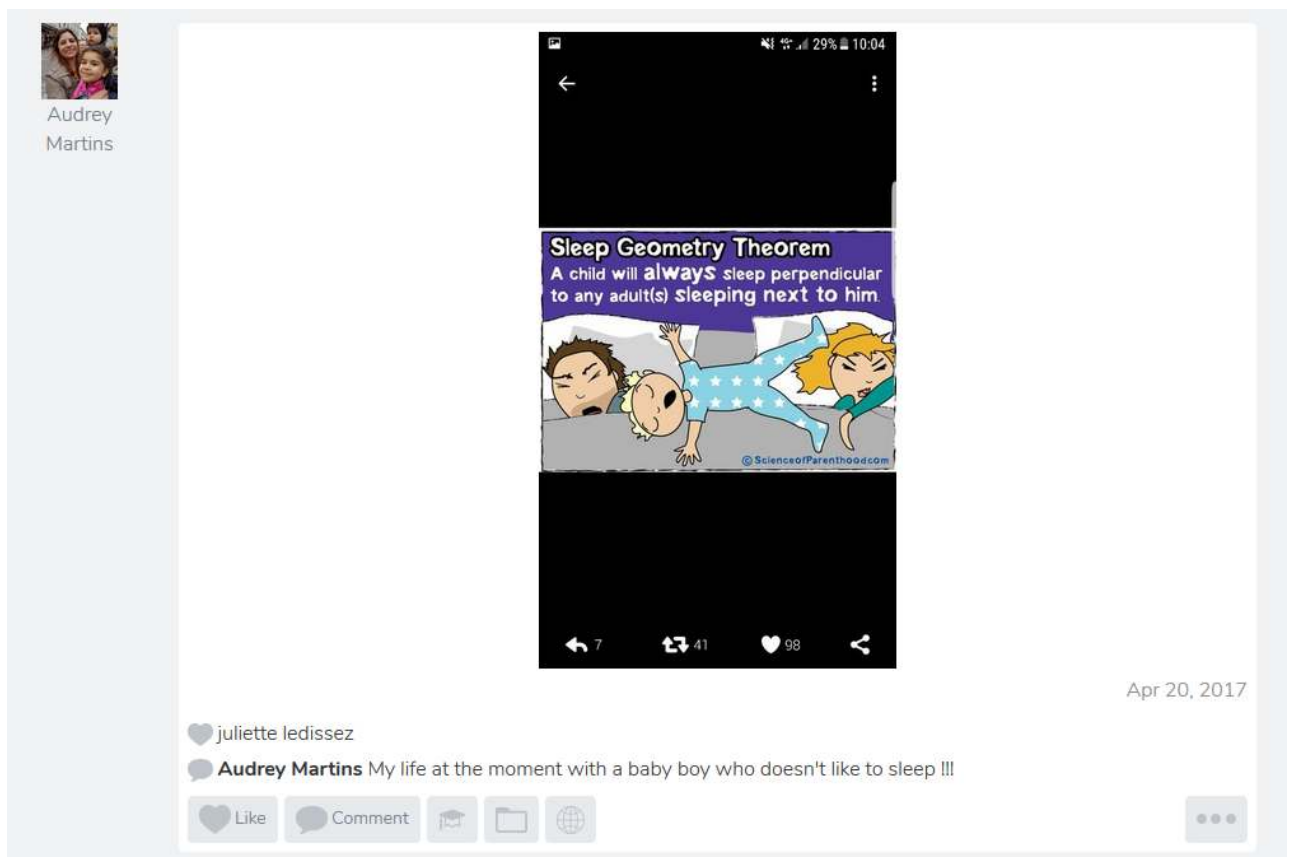

Extrait du carnet de bord rédigé sur l'application seesaw.com, montrant une image trouvée sur Internet sur ScienceofParenthood.com et insérée dans le message.

Plus loin encore de la justification d'un non-discours, le simple partage de la photo de ses chats par une étudiante, quasiment sans commentaire textuel, relève du socio-affectif, bien loin des craintes que l'on a pu voir formulées ci-dessus par certains sur les impératifs de calendrier ou de production écrite. 
54 À l'inverse, dans l'exemple suivant, où le discours fait une allusion aux pairs, l'étudiante arrive à concilier dans le même discours réponse à l'impératif de production écrite, réponse à l'impératif de pratique du numérique et tissage socio-affectif. Là encore, elle est bien au-delà d'un rapport de tension entre discours, non-discours et la justification de l'un ou de l'autre. Dans son message (ci-dessous), elle signale qu'elle a testé une ressource numérique de création de nuages de mots et que la forme choisie (un bébé, figure 13) est un clin d'œil à ses collègues enceintes :

Thème : Week 5!!!!!!!!!!!!!!!!!!!!!!!!!!!!!!! F. 3 mai 2017 14:03

[...] The chosen drawing is a little nod to pregnant student girlfriends, they will recognize each other!

Figure 13 : Un nuage de mots en forme de bébé

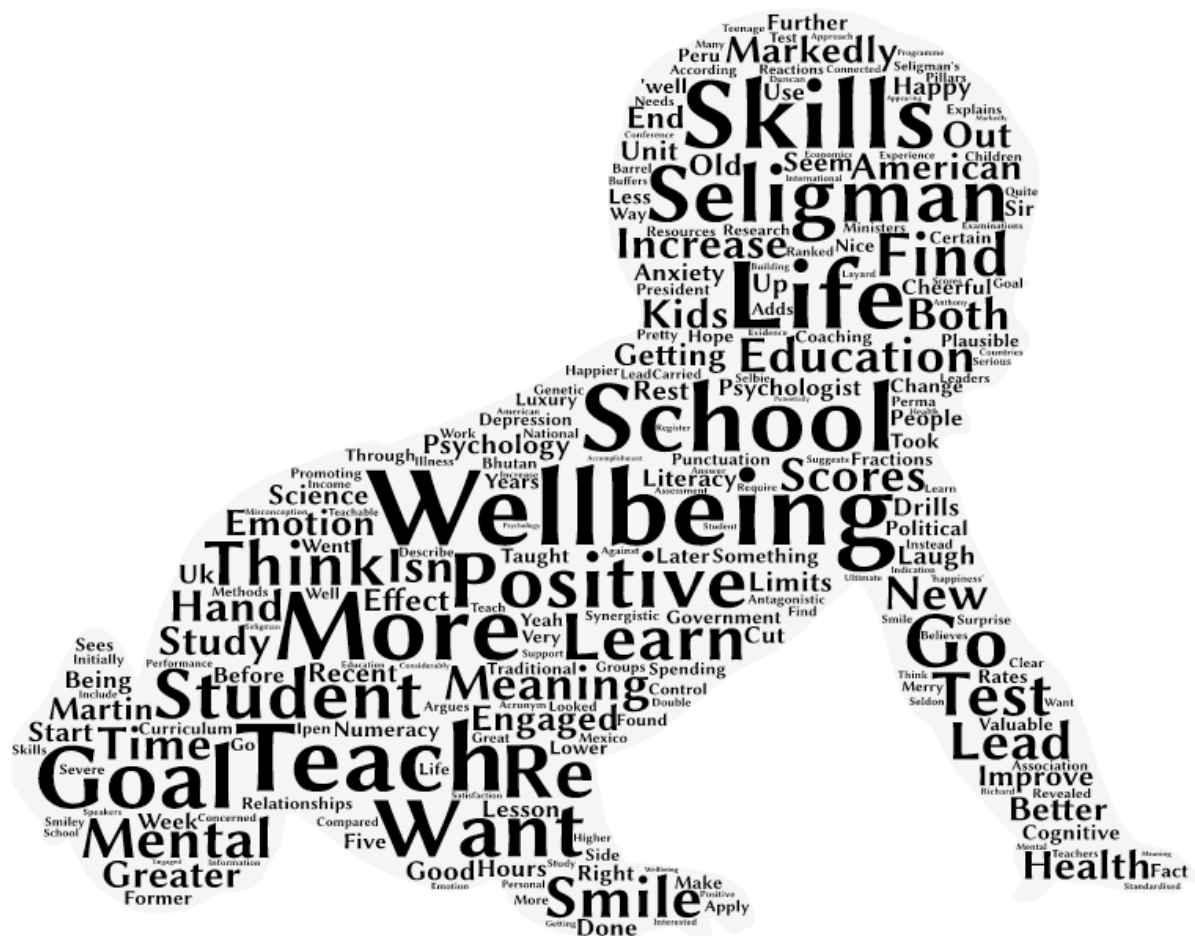

Extrait du forum montrant un nuage de mots en forme de bébé, réalisé à l'aide de l'application numérique wordart.com

A travers cette étude qualitative, nous avons vu apparaître que le silence numérique n'est pas anodin, et qu'au-delà d'être la simple trace de difficultés de participation de la part des étudiants, il induit des positionnements variés que nous détaillons en conclusion.

\section{Conclusion}

Ainsi, à travers une étude de corpus qualitative, ancrée sur une visualisation quantitative de la prégnance, parfois artificielle, de zones de silence numérique, nous avons vu apparaître, de l'autre côté de l'injonction de production langagière, un discours intégratif qui tisse parfois simultanément des aspects socio-affectifs et cognitifs. 
57 Notre interrogation portait sur les effets de la multiplication d'espaces de production langagière sur un forum d'apprentissage à distance, et sur les positionnements occasionnés par les injonctions à produire du langage, tels que décelables dans les discours.

58 Nous avons pu mettre en évidence des régimes de positionnement variés que nous récapitulons dans le tableau 1 ci-dessous.

Tableau 1. Régimes de positionnement par rapport à l'injonction de discours

\begin{tabular}{|l|l|}
\hline $\begin{array}{l}\text { Régimes de positionnement par rapport à } \\
\text { l'injonction de discours }\end{array}$ & $\begin{array}{l}\text { Absence de régime de positionnement par } \\
\text { rapport à l'injonction de discours }\end{array}$ \\
\hline Régime de la justification du non-discours & \\
\cline { 1 - 1 } Régime du refus du non-discours & \multirow{2}{*}{ Tissage socio-cognitif-affectif } \\
\cline { 1 - 1 } Régime du déni de non-discours & \\
\cline { 1 - 2 } Régime de l'anticipation du non-discours & \\
\hline
\end{tabular}

59 L'entrée par le «silence » nous a permis de rendre visibles certains aspects des tensions autour du discours et de l'absence de discours dans un environnement numérique. L'injonction de discours et la mise à disposition de lieux numériques multiples créent en creux du silence et génèrent un discours qui n'est parfois que référence au « silence » ou au non-discours. L'objectif est alors d'amener les étudiants à dépasser l'injonction de discours et l'injonction ressentie de justification pour arriver à un discours intégratif de la personne en interaction, qui mêle socio-affectif et cognitif. Cette relation apaisée au silence vise à dépasser les injonctions au cœur du didactique pour en faire des contraintes créatives.

\section{BIBLIOGRAPHIE}

Albero, Brigitte. « Une approche sociotechnique des environnements de formation. » Éducation et didactique, $\mathrm{n}^{\circ}$ 1, vol. 4, 2010, journals.openedition.org/educationdidactique/715. Consulté le 29 mars 2018.

Albero, Brigitte, et Arnim Kaiser. « Attitudes et préférences des usagers face à la formation ouverte et à distance. Les leçons d'une enquête. » Distances et savoirs, vol. 7, n 1, 2009, pp. 31-37, www.cairn.info/revue-distances-et-savoirs-2009-1-page-31.htm. Consulté le 23 avril 2018.

Audran, Jacques, et Claudine Garcin. « Apprendre en ligne, une question de participation? » Recherche et formation, $\mathrm{n}^{\circ} 68,2011$, journals.openedition.org/rechercheformation/1534. Consulté le 29 mars 2018. 
Bonenfant, Maude. «Le mème numérique : étude sémiotique des réseaux à partir des concepts de trace et d'indice. » RISCP, $\mathrm{n}^{\circ} 12,2014$. URL : communiquer.revues.org/1295. Consulté le 06 juin 2017.

Dimitracopoulou, Angélique, et Éric Bruillard. « Enrichir les interfaces de forums par la visualisation d'analyses automatiques des interactions et du contenu. » Revue STICEF, vol. 13, 2006, sticef.org. Consulté le 23 avril 2018.

Caranfa, Angelo. "Silence as the Foundation of Learning. Educational Theory. » Educational theory , n 54, 2004, pp. 211-230.

Cisel, Matthieu. Utilisations des MOOC : éléments de typologie. Thèse en Sciences de l'éducation, université Paris-Saclay, 2016.

Desmarais, Lise. «La persévérance dans l'enseignement à distance - Une étude de cas. » Alsic, $\mathrm{n}^{\circ}$ 1, vol. 3, 2000, document alsic_n05-rec5, mis en ligne le 15 juin 2000. URL : journals.openedition.org/alsic/. Consulté le 29 mars 2018.

Dussarps, Clément. «L'abandon en formation à distance. » Distances et médiations des savoirs, $\mathrm{n}^{\circ} 10$, 2015, journals.openedition.org/dms/1039. Consulté le 29 mars 2018

Dussarps, Clément et Didier Paquelin. « Pratiques sociales en formation à distance. » Netcom, $\mathrm{n}^{\circ}$ 28, vol. 3/4, 2014, journals.openedition.org/netcom/1815. Consulté le 29 mars 2018.

Eneau, Jérôme. « Formation d'adultes et dispositifs hybrides : comment 'faire confiance à distance'. » La confiance en questions. Paris, L’Harmattan, 2016, pp.143-164.

Grassin, Jean-François. « Un réseau sociopédagogique pour une formation en français langue étrangère (FLE). » Distances et médiations des savoirs, $\mathrm{n}^{\circ}$ 16, 2016, journals.openedition.org/ dms/1613. Consulté le 22 mai 2017.

Herring, Susan C. « Interactional coherence in CMC. » Journal of Computer-Mediated Communication, vol. $4, \mathrm{n}^{\circ} 2,1999$.

Holmer, T. (2008). « Discourse Structure Analysis of Chat Communication. » Language@Internet, vol. 5, http://www.languageatinternet.org/articles/2008/1633. Consulté le 22 mai 2017.

Huynh Kim Bang, Benjamin et Éric Bruillard (2005). « Vers une nouvelle interface de lecture pour des forums de discussion dédiés à des élaborations collectives. »Créer, jouer, échanger, actes de H2PTM'05, coordonné par Imad Saleh et Jean Clément Jean, Lavoisier, pp. 43-56.

Jézégou, Annie. «Créer de la présence à distance en e-learning. Cadre théorique, définition, et dimensions clés. » Distances et savoirs, vol. 8, n² 2, 2010, p. 257-274, www.cairn.info/revuedistances-et-savoirs-2010-2-page-257.htm. Consulté le 23 avril 2018.

Lejeune, Christophe. Manuel d'analyse qualitative. Analyser sans compter ni classer. De Boeck, 2014.

Lemke, Jay L. « Learning across multiple places and their chronotopes. » AERA 2004 Symposium: Spaces and boundaries of learning, 2004, San Diego, CA.

Teutsch, Philippe, et al. « Faciliter l'accès aux échanges en ligne et leur analyse, le cas de ViCoDiLi. » Revue STICEF, vol. 15, 2008, https://www.persee.fr/doc/ stice_1952-8302_2008_num_15_1_983. Consulté le 23 avril 2018.

Théviot, Anaïs. « Un silence numérique bavard. Controverses autour de l'interdiction de la propagande politique en ligne avant le vote. » Mots. Les langages du politique, $\mathrm{n}^{\circ}$ 103, 2013, journals.openedition.org/mots/21482. Consulté le 26 septembre 2017. 


\section{NOTES}

1. Notre proposition de traduction pour «shifting the mode of learning from discourse (knowing) to silence (unknowing) » Caranfa 227.

2. Les prénoms ont été modifiés.

3. Il s'agit d'identifier « les propriétés qui sont liées, qui varient ensemble, qui dépendent l'une de l'autre » (Lejeune 97).

\section{RÉSUMÉS}

À travers l'étude d'un corpus de productions écrites sur un forum d'enseignement à distance, nous mettons en lumière la tension entre injonction de discours et non-discours. Différents régimes donnent forme au positionnement dans cette dialectique : le régime du refus du nondiscours, le régime du déni du non-discours, le régime de l'anticipation du non-discours. Au-delà de cette tension, nous voyons émerger un rapport apaisé au silence avec des contributions d'apprenants en ligne qui mêlent le socio-affectif et le cognitif.

Through the study of a corpus of written productions on a distance teaching forum, we shed light on the tension between discourse injunction and non-discourse. Different regimes give shape to the positioning inside this dialectic: the non-discourse refusal regime, the non-discourse denial regime, the non-discourse anticipation regime. Beyond this tension, we see the emergence of an appeased relationship to silence with learners' online contributions that combine the socioaffective and the cognitive.

\section{INDEX}

Mots-clés : discours, forum, injonction, pratique de la langue, présence sociale, silence Keywords : discourse, forum, injunction, language practice, silence, social presence

\section{AUTEUR}

\section{JOSÉPHINE RÉMON}

Joséphine Rémon est actuellement maître de conférences à l'université de Lyon et travaille au sein du laboratoire ICAR (UMR5191). Après des études en linguistique et informatique, elle a été attachée linguistique à l'ambassade de France à Ottawa (Canada). Elle y découvre Internet et assiste à l'émergence de la Toile. Elle a consacré son doctorat à créer et implémenter des scénarios ludiques pour faire se rencontrer linguistique, apprentissage des langues et Internet. Elle enseigne l'anglais et le numérique à l'université Lyon2, aux étudiants anglicistes et en Master Sciences de l'éducation et Master Humanitaire. Ses recherches portent sur les traces de l'engagement et de la prise de risque pédagogiques et langagières dans les discours 